\title{
BMJ Open Global shortage of neonatal and paediatric antibiotic trials: rapid review
}

\author{
Georgina Thompson, ${ }^{1,2}$ Charlotte I Barker, ${ }^{1,3,4}$ Laura Folgori, ${ }^{1}$ Julia A Bielicki,,${ }^{1,5}$ \\ John S Bradley, ${ }^{6,7}$ Irja Lutsar, ${ }^{8}$ Mike Sharland ${ }^{1,4}$
}

To cite: Thompson $\mathrm{G}$, Barker Cl, Folgori L, et al. Global shortage of neonatal and paediatric antibiotic trials: rapid review. BMJ Open 2017;7:e016293. doi:10.1136/ bmjopen-2017-016293

- Prepublication history and additional material for this paper are available online. To view please visit the journal (http:// dx.doi.org/10.1136/bmjopen2017-016293).

Received 7 February 2017 Accepted 17 August 2017
CrossMark

For numbered affiliations see end of article.

Correspondence to Georgina Thompson; gethomps@sgul.ac.uk

\begin{abstract}
Objectives There have been few clinical trials (CTs) on antibiotics that inform neonatal and paediatric drug labelling. The rate of unlicensed and off-label prescribing in paediatrics remains high. It is unclear whether the current neonatal and paediatric antibiotic research pipeline is adequate to inform optimal drug dosing. Using the ClinicalTrials.gov registry, this review aims to establish the current global status of antibiotic CTs in children up to 18 years of age.
\end{abstract}

Methods Studies were identified using key word searches of the ClinicalTrials.gov registry and were manually filtered using prespecified inclusion/exclusion criteria.

Results 76 registered open CTs of antibiotics in children were identified globally; 23 (30\%) were recruiting newborns (only 8 (11\%) included preterm neonates), $52(68 \%)$ infants and toddlers, $58(76 \%)$ children and $54(71 \%)$ adolescents. The majority of registered trials were late phase $(10(15 \%)$ phase 3 and $23(35 \%)$ phase 4/pharmacovigilance). Two-thirds were sponsored by non-profit organisations, compared with pharmaceutical companies (50 (66\%) vs $26(34 \%)$, respectively). A greater proportion of non-profit funded trials were efficacy-based strategic trials $(n=34,68 \%)$, in comparison with industryled trials, which were most often focused on safety or pharmacokinetic data $(n=17,65 \%)$. Only 2 of the 37 antibiotics listed on the May 2016 Pew Charitable Trusts antibiotic development pipeline, currently being studied in adults, appear to be currently recruiting in open paediatric CTs.

Conclusions This review highlights that very few paediatric antibiotic CTs are being conducted globally, especially in neonates. There is a striking disparity noted between antibiotic drug development programmes in adults and children.

\section{INTRODUCTION}

Widespread unlicensed and off-label prescribing in paediatrics persists-as high as $11.4 \%$ and $46.5 \%$, respectively-yet the paucity of clinical research involving children that is conducted to inform optimal drug dosing, licensing and labelling remains a problem. ${ }^{1}$ For certain medicines, drug efficacy can be extrapolated from adult data provided that the pathology and drug exposure are the same or sufficiently similar in children as in adults. ${ }^{2} 3$ Although differences
Strengths and limitations of this study

A narrative literature review of registered clinical trials in children.

- Explicit reproducible methodology.

- Search strategy limited to ClinicalTrials.gov and EudraCT. Entirety of clinical research in this field might not have been captured.

- Search strategy was limited to open clinical trials. Active but not yet recruiting trials were not captured.

in drug pharmacokinetics (PK) in neonates and children can lead to adverse reactions that are not seen in adult populations, these are very rare. ${ }^{4}$ Suboptimal antibiotic dosing, including underdosing and overdosing, can lead to toxicity and treatment failure and may drive antimicrobial resistance by encouraging selection pressures on drug-resistant strains of bacteria. ${ }^{5}$

Since antibiotics are the most commonly prescribed medicines in children, it is important to maximise our understanding of their PK profiles to help determine optimal drug dosing and ultimately to improve outcomes. ${ }^{6}$ In the last decade, several initiatives have been established to encourage paediatric medicines research, bridging the gap between adult and paediatric drug development plans. Such initiatives include the Paediatric Regulation (Paediatric Investigation Plans (PIPs), introduced by the European Medicines Agency (EMA), ${ }^{7}$ and Pediatric Study Plans, by the US Food and Drug Administration (FDA)). ${ }^{8}$ Despite this, there have been few advances in antibiotic development for this population. ${ }^{9}$

The global status of clinical research on antibiotics in paediatrics is unknown. Using registered records of clinical trials (CTs) on ClinicalTrials.gov, this review aims (1) to summarise the current global status of registered antibiotic research in children and neonates and (2) to stimulate discussion and collaboration among the relevant 
258 records excluded on abstract:

40 antiseptics

34 antifungal

84 antiprotozoal

81 antiviral

19 other

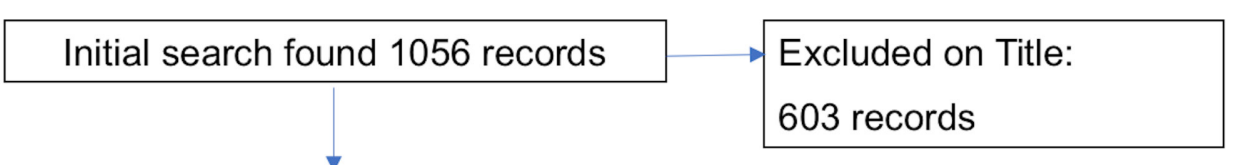

453 records investigating one or more antiinfective agents in children under 18 years of age

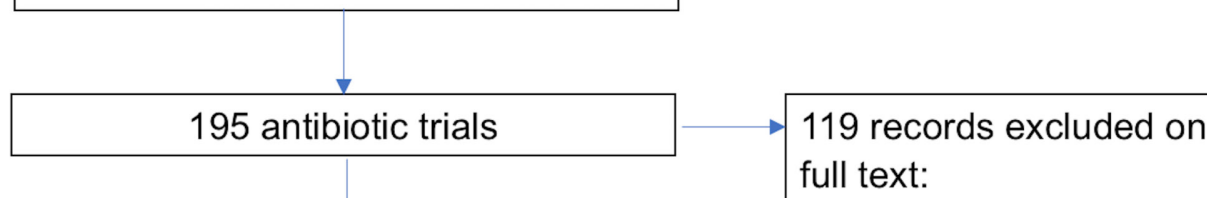
full text:

12 Tuberculosis

76 antibiotic trials included in review
2 Malaria

15 Cystic fibrosis

2 HIV

8 Febrile neutropaenia

12 Topical

6 Inhalational

38 Prophylaxis

24 Alternative use

Figure 1 Flow chart. Clinical trial selection process.

stakeholders on the neglect of antibiotic research in children.

\section{METHODS}

\section{Data sources}

The ClinicalTrials.gov registry (last accessed 8 November 2016) is an international platform for the registration of CTs. It is a web-based registry, developed in 2000 by the National Institutes of Health and the FDA, to which trials from 50 states and 163 countries around the world are registered. Information provided for each trial is updated periodically by the trial's sponsoring organisation. The database has a specific child filter, which uses a key word paradigm to select all registered trials recruiting patients/ participants up to 18 years of age.

\section{Study selection}

Our records were identified using suitable key word searches, in which the final search terms were (antimicrobial* OR antibiotic* OR anti-infective agent*) AND Child AND Open Studies. All identified trials were filtered manually using inclusion and exclusion criteria. Interventional and observational trials on antimicrobials recruiting children up to 18 years of age were considered eligible for inclusion. No specific temporal filter was applied since only open or ongoing clinical trials were of interest. The following records, which were not investigating one or more antibiotics, were excluded: trials of antiseptics, antifungals, antiprotozoals, antivirals and probiotics or prebiotics. The following records, which were investigating an antibiotic, were excluded: trials involving tuberculosis, malaria, cystic fibrosis, HIV, febrile neutropenic patients, topical or inhalational treatments and prophylactic antibiotics or records investigating alternative use for antibiotics (eg, as an anti-inflammatory agent).

The searches were conducted by GT. All eligible records were identified via manual filtering by GT, CIB, LF and MS, and any disagreements regarding inclusion and exclusion of records were resolved through discussion.

\section{Data extraction}

The following information was collected from the included records: unique ClinicalTrials.gov identifier (NCT number), recruitment status, study design, trial phase, study sponsor, age group and sex eligibility, clinical indication, geographic region of recruitment, antibiotic being investigated and endpoint classification. Outcomes were categorised as safety, efficacy or PK. Economic setting (based on geographic region of recruitment) was classified using The World Bank classification to differentiate between low-income countries (LICs), lower income and middle-income countries (LMICs), upper middle-income Countries (UMICs) and high-income countries (HICs). ${ }^{10}$

The specific class of antibiotic studied in each trial was identified and classified using the WHO Anatomical, Therapeutic, and Chemical classification and Defined 
Table 1 Characteristics of clinical trials

\begin{tabular}{|c|c|c|}
\hline Characteristic & Category & $\begin{array}{l}\text { Number of } \\
\text { studies, } \mathrm{n}(\%)\end{array}$ \\
\hline \multirow[t]{5}{*}{ Age group } & Preterm neonates & $1(1)^{*}$ \\
\hline & Neonates (total) & $23(30)$ \\
\hline & Infants and toddlers & $52(68)$ \\
\hline & Children & $58(76)$ \\
\hline & Adolescents & $54(71)$ \\
\hline \multirow[t]{2}{*}{ Recruitment status } & Recruiting & $63(83)$ \\
\hline & Not yet recruiting & $13(17)$ \\
\hline \multirow[t]{2}{*}{ Study design } & Interventional & $66(87)$ \\
\hline & Observational & $10(13)$ \\
\hline \multirow[t]{7}{*}{ Trial phase $†$} & Phase 1 & $10(15)$ \\
\hline & Phases 1-2 & $1(2)$ \\
\hline & Phase 2 & $9(14)$ \\
\hline & Phases 2-3 & $3(5)$ \\
\hline & Phase 3 & $10(15)$ \\
\hline & Phase 4 & $23(39)$ \\
\hline & Not specified & $10(15)$ \\
\hline \multirow[t]{2}{*}{ Sponsor } & Industry & $26(34)$ \\
\hline & Non-profit & $50(66)$ \\
\hline \multirow[t]{6}{*}{ Geographic region } & Africa & $8(11)$ \\
\hline & Asia & $16(21)$ \\
\hline & Europe & 22 (29) \\
\hline & Latin America & $6(8)$ \\
\hline & North America & $34(45)$ \\
\hline & Oceania & $5(7)$ \\
\hline \multirow[t]{9}{*}{ Antibiotic class } & J01A Tetracycline & $4(5)$ \\
\hline & $\begin{array}{l}\text { J01C Beta-lactam, } \\
\text { penicillin }\end{array}$ & $25(33)$ \\
\hline & J01D Other beta-lactam & $22(29)$ \\
\hline & $\begin{array}{l}\text { J01E Sulfonamides and } \\
\text { trimethoprim }\end{array}$ & $7(9)$ \\
\hline & $\begin{array}{l}\text { J01F Macrolide, } \\
\text { lincosamide, } \\
\text { streptogramin }\end{array}$ & $14(18)$ \\
\hline & J01G Aminoglycoside & $2(3)$ \\
\hline & J01M Quinolone & $8(11)$ \\
\hline & $\begin{array}{l}\text { J01X Other antibiotic } \\
\text { classes } \ddagger\end{array}$ & $21(28)$ \\
\hline & J01 Not specified & $2(3)$ \\
\hline
\end{tabular}

Totals for age group, geographic region and antibiotic class do not add up to total number of clinical trials (76) as some trials contributed to more than one subgroup.

*Seven further trials mentioned inclusion of preterm babies in the inclusion criteria.

†Trial phase $\%$ based on percentage of interventional trials. łJ01X includes glycopeptides, polymyxins, imidazole and nitrofuran derivatives.

Daily Dose (ATC/DDD) index. ${ }^{11}$ To investigate whether novel antibiotics are being studied in paediatric populations, the antibiotics currently studied in children
Table 2 Clinical trial endpoint classification of identified clinical trials stratified by trial sponsor

\begin{tabular}{lccl}
\hline $\begin{array}{l}\text { Endpoint } \\
\text { classification }\end{array}$ & Industry & Non-profit & Total (\%) \\
\hline Efficacy & 9 & 34 & $43(57)$ \\
Safety & 10 & 2 & $12(16)$ \\
PK & 7 & 14 & $21(28)$ \\
\hline
\end{tabular}

Endpoint classification determined by planned primary outcomes. PK, pharmacokinetics.

were compared with the Pew Charitable Trusts Antibiotics Currently in Clinical Development pipeline, which identifies novel antibiotics currently under development for the US market. $^{12}$

\section{RESULTS}

Our search identified 1056 records. Six hundred and three records were excluded on title because they were studies not involving an antimicrobial. Four hundred and fifty-three records were investigating one or more antimicrobials and recruiting children below 18 years of age. Among the 195 trials investigating antibiotics, 76 fulfilled our inclusion and exclusion criteria and were included in the final analysis. Reasons for exclusion are summarised in figure 1. Details of included studies can be found in online supplementary file 1 .

All 76 CTs identified were open as of 8 November 2016, and $63(83 \%)$ of these were recognised as recruiting participants on this date (table 1 ). All trials stated recruitment of both male and female participants.

\section{Age group}

Twenty-three of the 76 trials (30\%) were recruiting newborns (0-28 days). One of these 23 trials focused solely on recruiting preterm newborns (a further 7 CTs mentioned inclusion of preterm newborns in inclusion criteria). Of the remaining records, 52 (68\%) were recruiting infants and toddlers (28 days-23 months), 58 (76\%) children (2-11 years) and $54(71 \%)$ adolescents (11-18 years). Twenty-nine (38\%) trials did not focus solely on the recruitment of children or neonates, with age ranges also spanning across adult populations.

\section{Study type}

Interventional trials were most frequently identified $(n=66,87 \%)$ with only $10(13 \%)$ observational trials noted. Of the interventional trials, the majority were in the later stages of development; $10(13 \%)$ in phase 1, 1 (2\%) between phases 1 and 2, $9(14 \%)$ in phase 2, $3(5 \%)$ between phase 2 and phase 3, $10(15 \%)$ in phase 3 and $23(35 \%)$ in phase 4 . In $10(15 \%)$ cases, a trial phase was not specified.

\section{Sponsor and endpoint classification}

Fifty trials $(66 \%)$ were sponsored by non-profit organisations (being university, hospital or government funded), 
Table 3 Clinical indication of identified clinical trials stratified by age group being recruited

\begin{tabular}{lllllll}
\hline & & \multicolumn{5}{c}{ Age group } \\
\cline { 3 - 7 } Indication & Total (\%) & Preterm neonates & Neonates* & Infants and toddlers & Children & Adolescents \\
\hline Unspecified bacterial infection & $18(24)$ & - & 11 & 15 & 15 & 14 \\
Lower respiratory tract infection & $12(16)$ & 1 & 3 & 12 & 13 & 5 \\
Sepsis & $11(14)$ & - & 6 & 9 & 9 & 11 \\
Upper respiratory tract & $8(11)$ & - & 1 & 5 & 9 & 7 \\
Intra-abdominal infection & $8(11)$ & - & 3 & 5 & 5 & 7 \\
Urinary tract infection & $7(9)$ & - & 2 & 6 & 7 & 6 \\
Skin and soft tissue infection & $6(8)$ & - & 3 & 4 & 4 & 8 \\
CNS infection & $3(4)$ & - & 1 & 4 & 4 & 4 \\
Bone and joint Infection & $1(1)$ & - & - & 1 & 1 & 1 \\
& & 1 & 30 & 61 & 67 & 63
\end{tabular}

Age group totals do not add up to total number of clinical trials (76) as some trials contributed to more than one age group.

*Total number of preterm and term neonates.

and 26 trials were sponsored by industry (34\%). The endpoint classification of the majority of trials $(n=43$, $57 \%$ ) was reported as efficacy (table 2). A greater proportion $(n=34,68 \%)$ of non-profit studies measured the efficacy of the drugs as the primary endpoint, with less emphasis on collection of PK or safety data $(n=16,32 \%)$. In comparison, pharmaceutical-led trials focused on early PK and safety studies over the drug's efficacy $(n=17,65 \%$ vs $\mathrm{n}=9,35 \%$, respectively).

\section{Geographic region}

The most frequently recruiting geographic region was North America $(\mathrm{n}=34,45 \%)$. Twenty-two $(29 \%)$ trials recruiting in Europe and $16(21 \%)$ in Asia were identified, $6(8 \%)$ in Latin America, $8(11 \%)$ in Africa and $5(7 \%)$ in Oceania. Most trials were recruiting in HICs $(\mathrm{n}=54,71 \%)$, with fewer trials recruiting in LICs $(\mathrm{n}=4$, $5 \%)$, LMICs $(\mathrm{n}=4,5 \%)$, UMICs $(\mathrm{n}=11,14 \%)$ or a combination of UMICs and HICs $(\mathrm{n}=3,4 \%)$.

\section{Indication}

The most common treatment indications investigated were lower respiratory tract infection $(\mathrm{n}=12,16 \%)$ and sepsis $(\mathrm{n}=11,14 \%)$, followed by upper respiratory tract infection $(n=8,11 \%)$, intra-abdominal infection (IAI) $(\mathrm{n}=8,11 \%)$, urinary tract infection (UTI) $(\mathrm{n}=7,9 \%)$, complicated skin and soft tissue infection (cSSTI) $(\mathrm{n}=6$, $8 \%)$, central nervous system (CNS) infection $(n=3,4 \%)$ and bone and joint infection $(\mathrm{n}=1,1 \%)$ (table 3$)$.

\section{Antibiotic class}

The majority of antibiotics being investigated were beta-lactams $(n=47,62 \%)$, followed by other antibiotic classes (J01X, including vancomycin, telavancin and dalbavancin) $(\mathrm{n}=21,28 \%)$. Macrolides or lincosamides (J01F) were the next most commonly studied antibiotic classes $(n=14,18 \%)$. Very few trials were investigating of tetracyclines (J01A) $(\mathrm{n}=4,5 \%)$, sulphonamides and trimethoprim (J01E) $(\mathrm{n}=7,9 \%)$, aminoglycosides (J01G) $(\mathrm{n}=2,3 \%)$ or quinolones $(\mathrm{J} 01 \mathrm{M})(\mathrm{n}=8,11 \%)$. Two CTs (3\%) did not specify the class of antibiotic being investigated. Sixteen $(21 \%)$ trials were investigating more than one antibiotic; these trials counted towards more than one J01 category. The breakdown of J01 categories, as per WHO ATC/DDD classification, ${ }^{11}$ is described in table 1.

\section{Antibiotic pipeline}

Of the 37 antibiotics listed in the May 2016 edition of the Pew Charitable Trusts Antibiotic Pipeline (last accessed 10 June 2016), ${ }^{12}$ as noted by the EMA Opinions and Decisions on Pediatric Investigation Plans, five had an agreed PIP: imipenem/cilastatin+relebactam, cadazolid, carbavance (meropenem+vaborbactam), eravacycline and solithromycin. ${ }^{8}$ As of 8 November 2016, our search found that only 2 of these 37 antibiotics listed (carbavance and solithromycin) were being investigated in 1 and 2 ongoing CTs in paediatric patients, respectively (table 4). A PIP was agreed for carbavance in 2015 for treatment of Gram-negative infections, and for solithromycin in 2016 for the treatment of gonococcal infection, and later for treatment of anthrax, tularaemia and bacterial pneumonia. PIPs were agreed in 2015 for treatment of UTI and complicated IAI with eravacycline, and in 2016 for treatment of Clostridium difficile infection with cadazolid and of Gram-negative bacterial infection with imipenem/ cilastin+relebactam. ${ }^{13}$ Despite this, we could not identify any registered trials of these antibiotics in our search.

\section{DISCUSSION}

Our search identified 76 clinical trials investigating one or more antibiotics recruiting children between 0 and 18 years of age. This is low in comparison with the number of ongoing trials in adults, despite children representing around a quarter of the global population. ${ }^{14}{ }^{15}$ A review of completed CTs in the USA between 2000 and 2010 identified a total of 4078 adult trials 


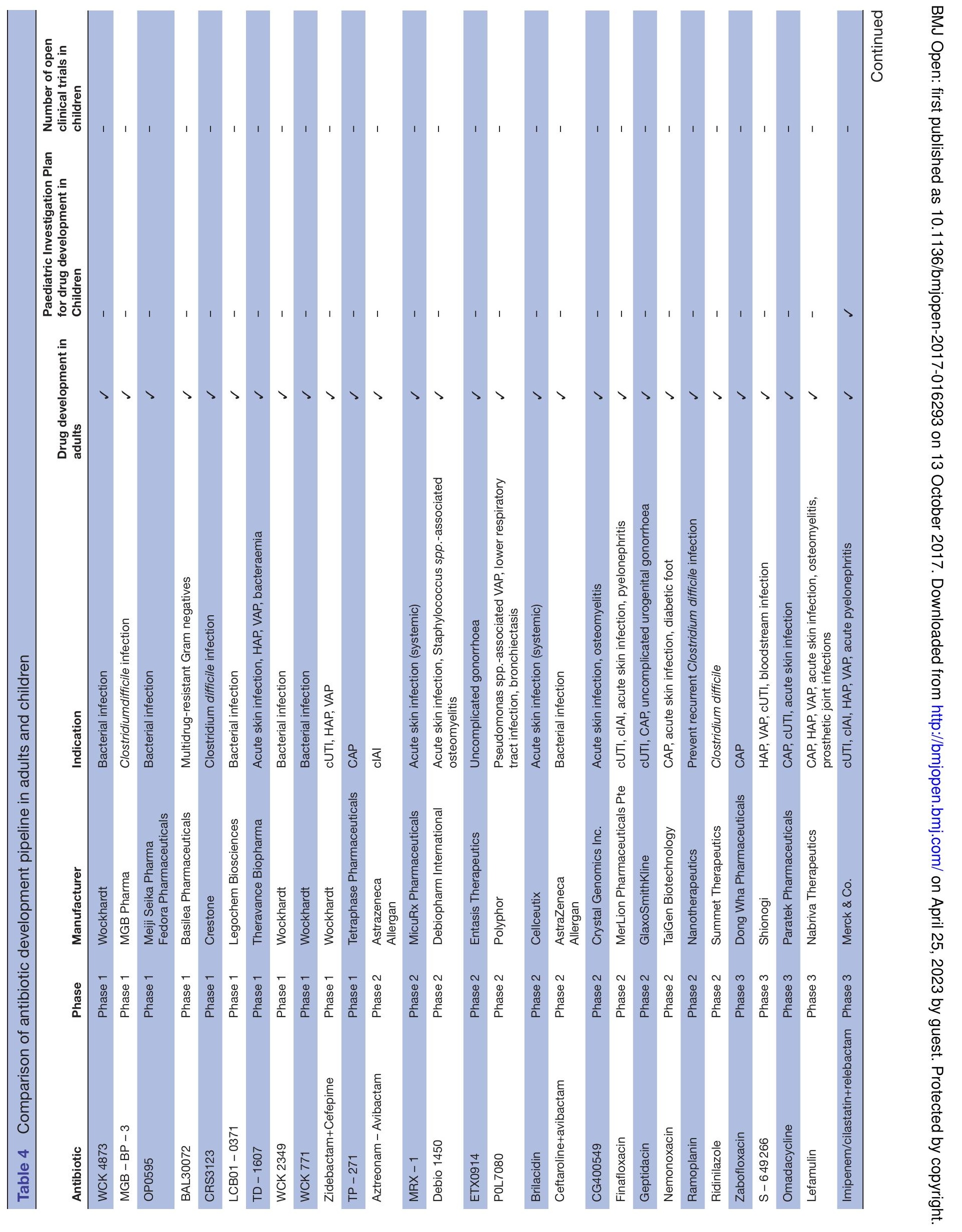


compared with just 294 that had recruited children. ${ }^{16}$ In our study, the lack of trials recruiting neonates is striking. Just 23 of the 76 trials identified were recruiting neonates and remarkably just 8 CTs globally were recruiting preterm neonates. There are broadly two types of paediatric trials conducted globally: (1) pharmaceutical-led phase $1 / 2 \mathrm{PK}$ and safety trials $(\mathrm{n}=17)$ being conducted in the HIC setting, and (2) investigator-led, often pragmatic, late phase efficacy trials in the LMIC setting $(n=34)$. Amongst these a greater proportion of ongoing trials are sponsored by non-profit organisations over industry $(66 \%$ compared with 34\%). As of April 2016, there were 17 antibiotic PIPs agreed by the EMA, ${ }^{8}$ covering a range of indications, most commonly cSSTI, complicated IAI (cIAI) and complicated UTI (cUTI) ${ }^{13}$ In contrast, treatments for respiratory and systemic infections, the most common clinical indications for antibiotics in paediatrics, are not currently being evaluated. ${ }^{14}$ Thirty-seven antibiotics are currently being developed in adults, yet to our knowledge, just two of these are being studied in children. Some classes of antibiotics that may be of higher risk for children (tetracyclines and fluoroquinolones) may not be pursued as aggressively for paediatric approvals due to well-recognised issues of toxicity, particularly when safer alternatives are widely available. Given that Gram-negative sepsis is a growing problem in neonates, with a significant increase in the proportion of multiresistant Gram-negative pathogens, ${ }^{15}$ the substantial lag time between the submission date (for PIP or waiver) and declared completion date of PK studies in adults is a real concern, although there is value in generating substantial safety data in adults prior to exposing children and newborns to potentially toxic new agents.

In 2013, a similar review of European paediatric clinical trials identified 31 trials of antibiotics approved for adults by the EMA in 2000 that were recruiting children in Europe (compared with the 22 trials we found recruiting in Europe). They included both published and ongoing trials, which likely accounts for the higher number of trials reported. They similarly found a very small proportion of neonatal trials (2 of 31 ), as well as a greater proportion of efficacy-based trials. ${ }^{9}$ A review of interventional trials registered with ClinicalTrials. gov between 2007 and 2010 found that only $17 \%$ had recruited children below 18 years of age. ${ }^{17} \mathrm{~A}$ similar review of antimicrobial CTs conducted in the USA between 2000 and 2012 reported that just 5\% had recruited only children compared with $74 \%$ that recruited only adults, and that, as we have found, the trials were sponsored primarily by non-profit organisations ( $60 \%$ vs $30 \%$ by industry) ${ }^{14}$ In our search of global trials, $39 \%$ of registered CTs reported collection of PK data in comparison with a 2009 paediatric PK research review that reported $24 \%$ of registered CTs would be collecting PK data. ${ }^{18}$

The search strategy used has some limitations. Since the search was limited to clinical trials registered with 
ClinicalTrials.gov, it is possible other open or ongoing trials registered with alternative platforms (eg, the WHO International Clinical Trials Registry Platform) will have been missed. Together with ClinicalTrials.gov, these could help to establish the entirety of current clinical research in this field. We did, however, search EudraCT and found no further studies beyond those captured on ClinicalTrials. gov. Our search was also limited to open CTs, thereby missing active but not yet recruiting trials and those that had already closed to recruitment. The information recorded for each trial registered with ClinicalTrials.gov is updated by the trial investigators, and therefore relies on them to periodically update the registry. Occasionally, information such as recruitment status might not be updated in real time.

Concerns around the growing threat of antimicrobial resistance have prompted several new initiatives. In 2016, the EMA published a draft concept paper to propose the development of an addendum to the guideline on the evaluation of new antibacterial products for treatment of bacterial infections in children. ${ }^{19}$ At the same time, the Clinical Trials Transformation Initiative is currently focused on the identification of key barriers in the conduct of paediatric antibacterial CTs, which hamper their successful implementation into clinical practice. ${ }^{20}$ Recent evidence states that, overall, antibiotic CTs make up less than $1 \%$ of all registered paediatric CTs and that trial completion is slow, with an average time to completion of around 2 years. ${ }^{21}$ There are specific areas where the design and conduct of paediatric antibiotic CTs can be harmonised and simplified, such as the standardisation of the inclusion and exclusion criteria for specific clinical infection syndromes, and improved bridging of safety and efficacy data from other age groups; these advances could improve trial conduct and efficiency in children. $^{22}$

\section{CONCLUSIONS}

This review highlights that very few paediatric antibiotic CTs are being conducted globally, particularly in neonates. There is a marked disparity between antibiotic drug development programmes in adults and children. Many issues contribute to the difficulties in conducting paediatric antibiotic clinical trials. The lack of regulatory guidance, vulnerability of this population, issues with informed consent and assent and lack of research-experienced hospital personnel all present challenges in study design, delivery and recruitment. Delays in the initial start-up of CTs in paediatrics due to paediatric-specific protocol issues and complicated ethical approval continue to discourage both academic and pharmaceutical interest. The limited data presented here suggest that the dismal state of paediatric antibiotic research continues. Earlier collaboration between global academic research networks and pharmaceutical companies is now vital to accelerate progress.

\section{Author affiliations}

${ }^{1}$ Paediatric Infectious Diseases Research Group, Institute for Infection and Immunity, St George's University of London, London, UK

${ }^{2}$ University of Exeter Medical School, University of Exeter, Exeter, UK

${ }^{3}$ Inflammation, Infection and Rheumatology Section, UCL Great Ormond Street Institute of Child Health, London, UK

${ }^{4}$ Paediatric Infectious Disease, St George's University Hospitals NHS Foundation Trust, London, London, UK

${ }^{5}$ Department of Paediatric Pharmacology and Pharmacometrics, University of Basel Children's Hospital, Basel, Switzerland

${ }^{6}$ Department of Pediatrics, School of Medicine, University of California, San Diego, California, USA

${ }^{7}$ Division of Infectious Disease, Rady Children's Hospital San Diego, San Diego, California, USA

${ }^{8}$ Department of Medical Microbiology, University of Tartu, Tartu, Estonia

Contributors MS, CIB, GT, LF and JAB designed the study. Searches were conducted by GT. Eligible records were identified via manual filtering by GT, CIB, LF and MS. IL and JSB commented on study design and assisted with drafting the manuscript.

Funding This research received no specific grant from any funding agency in the public, commercial or not-for-profit sectors. CIB was funded as a clinical research fellow by the Global Research in Paediatrics (GRiP) Network of Excellence, part of the European Union's Seventh Framework Programme for research, technological development and demonstration (FP7/2007-2013, grant agreement number 261060).

\section{Competing interests None declared.}

Provenance and peer review Not commissioned; externally peer reviewed.

Data sharing statement Full dataset is available on request.

Open Access This is an Open Access article distributed in accordance with the Creative Commons Attribution Non Commercial (CC BY-NC 4.0) license, which permits others to distribute, remix, adapt, build upon this work non-commercially, and license their derivative works on different terms, provided the original work is properly cited and the use is non-commercial. See: http://creativecommons.org/ licenses/by-nc/4.0/

(c) Article author(s) (or their employer(s) unless otherwise stated in the text of the article) 2017. All rights reserved. No commercial use is permitted unless otherwise expressly granted.

\section{REFERENCES}

1. Corny J, Lebel D, Bailey B, et al. Unlicensed and off-label drug use in children before and after pediatric governmental initiatives. J Pediatr Pharmacol Ther 2015;20:316-28.

2. European Medicines Agency. Concept paper on extrapolation of efficacy and safety in medicine development. http://www.ema. europa.eu/docs/en_GB/document_library/Scientific_guideline/2013/ 04/WC500142358. pdf (accessed May 2016).

3. Dunne J, Rodriguez WJ, Murphy MD, et al. Extrapolation of adult data and other data in pediatric drug-development programs. Pediatrics 2011;128:e1242-9. http://pediatrics.aappublications.org/ content/early/2011/10/20/peds.2010-3487.abstract (accessed June 2016).

4. Kearns GL, Abdel-Rahman SM, Alander SW, et al. Developmental pharmacology-drug disposition, action, and therapy in infants and children. N Engl J Med 2003;349:1157-67.

5. Theuretzbacher U, Van Bambeke F, Cantón R, et al. Reviving old antibiotics. Internet J Antimicrob Chemother 2015. http://jac. oxfordjournals.org/content/early/2015/06/10/jac.dkv157.abstract (accessed June 2016)

6. van der Meer JW, Gyssens IC. Quality of antimicrobial drug prescription in hospital. Clin Microbiol Infect 2001;7(Suppl 6):12-15.

7. European Medicines Agency. Ema opinions and decisions on paediatric investigation plans. 2016. http://www.ema.europa.eu/ ema/index.jsp?curl=pages/medicines/landing/pip_search.jsp\&mid= WC0b01ac058001d129 (accessed June 2016).

8. Guidance for industry. Pediatric study plans: content of and process for submitting initial pediatric study plans and amended initial pediatric study plans. http://www.fda.gov/downloads/drugs/ guidancecomplianceregulatoryinformation/guidances/ucm360507. pdf (accessed June 2016). 
9. Garazzino S, Lutsar I, Bertaina C, et al. New antibiotics for paediatric use: a review of a decade of regulatory trials submitted to the European Medicines Agency from 2000-why aren't we doing better? Int J Antimicrob Agents 2013;42:99-118.

10. The World Bank. The World Bank list of economies. 2015. http:// siteresources.worldbank.org/DATASTATISTICS/Resources/CLASS. XLS (accessed Oct 2016).

11. The World Health Organisation. WHO ATC/DDD Index. 2016. http:// www.whocc.no/atc_ddd_index/ (accessed Mar 2016).

12. The Pew Charitable Trusts. Antibiotics Currently in Clinical Development. 2016. http://www.pewtrusts.org/ /media/assets/2016/ 05/antibiotics-currently-in-clinical-development.pdf?la=en (accessed May 2016)

13. Lutsar I. Often neglegted: paediatric drug development - a regulatory and clinical view. Amsterdam, Netherlands, 2016. (S219 Symposium lecture).

14. Stockmann C, Sherwin CM, Ampofo K, et al. Characteristics of antimicrobial studies registered in the USA through ClinicalTrials.Gov. Int $J$ Antimicrob Agents 2013;42:161-6.

15. Versporten A, Bielicki J, Drapier N, et al. The Worldwide Antibiotic Resistance and Prescribing in European Children (ARPEC) point prevalence survey: developing hospital-quality indicators of antibiotic prescribing for children. J Antimicrob Chemother 2016;71:1106-17.
16. Bielicki JA, Lundin R, Sharland M. Antibiotic Resistance Prevalence in Routine Bloodstream Isolates from Children's Hospitals Varies Substantially from Adult Surveillance Data in Europe. Pediatr Infect Dis J 2015;34:734-41.

17. Califf RM, Zarin DA, Kramer JM, et al. Characteristics of clinical trials registered in ClinicalTrials.gov, 2007-2010. JAMA 2012;307:1838-47.

18. Viergever RF, Rademaker CM, Ghersi D. Pharmacokinetic research in children: an analysis of registered records of clinical trials. BMJ Open 2011:1:e000221.

19. European Medicines Agency. Concept paper on an addendum to the guideline on the evaluation of medicinal products indicated for treatment of bacterial infections to address paediatric-specific clinical data requirements. http://www.ema.europa.eu/docs/en_GB/ document_library/Scientific_guideline/2016/04/WC500205026.pdf (accessed July 2016).

20. Clinical Trials Transformation Initiative. https://www.ctti-clinicaltrials. org/projects/pediatric-trials (accessed July 2016).

21. Clinical Trials Transformation Initiative. AACT database. https://www. ctti-clinicaltrials.org/aact-database (accessed Jan 2017).

22. Folgori L, Bielicki J, Ruiz B, et al. Harmonisation in study design and outcomes in paediatric antibiotic clinical trials: a systematic review. Lancet Infect Dis 2016;16:e178-89. 